\title{
Ventricular Arrhythmias in Endurance Athletes. Are Heart Rate Monitors Suitable Tools for their Diagnostics?
}

ISSN: 2577-1914

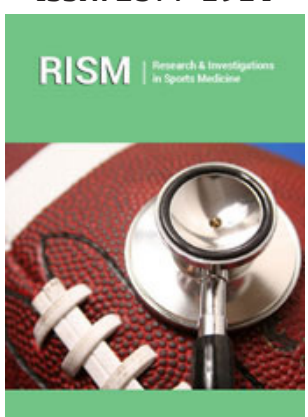

*Corresponding author: Robert Gajda, Center for Sports Cardiology at the Gajda-Med Medical Center, Pułtusk, Poland

Submission: 眥 October 30, 2019

Published: 留 November 27, 2019

Volume 5 - Issue 5

How to cite this article: Robert Gajda, Wojciech Drygas. Ventricular Arrhythmias in Endurance Athletes. Are Heart Rate Monitors Suitable Tools for their Diagnostics?. Res Inves Sports Med. 5(5). RISM.000622.2019.

DOI: $10.31031 /$ RISM.2019.05.000622

Copyright@ Robert Gajda, This article is distributed under the terms of the Creative Commons Attribution 4.0 International License, which permits unrestricted use and redistribution provided that the original author and source are credited.

\author{
Robert Gajda ${ }^{1 *}$ and Wojciech Drygas ${ }^{2,3}$ \\ ${ }^{1}$ Center for Sports Cardiology at the Gajda-Med Medical Center, Poland \\ ${ }^{2}$ The Cardinal Stefan Wyszyński Institute of Cardiology, Poland \\ ${ }^{3}$ Department of Preventive Medicine, Medical University of Lodz, Poland
}

\begin{abstract}
Keywords: Arrhythmia; Ventricular tachycardia; Endurance athletes; Heart rate monitors (HRMs); Exercise
\end{abstract}

Abbreviations: HRMs: Heart Rate Monitors; DGE: Delayed Gadolinium Enhancement; MRI: Magnetic Resonance Imaging; VPB: Ventricular Premature Beats; VT: Ventricular tachycardia; RVOT: Right Ventricular Outflow Tract; ARVC: Arrhythmogenic Right Ventricular Cardiomyopathy; ICD: Implantable Cardioverter Defibrillator; SCD: Sudden Cardiac Death; CAH: Care of the Athletic Heart, HRV: Heart Rhythm Variability

\section{Ventricular Arrhythmias}

Arrhythmias in competitive athletes are not rare. The prevalence of ventricular arrhythmias in people playing sports is not easy to estimate. First reports come from the 80 s-90s of the 20th century and there are no contemporary studies concerning the frequency of ventricular arrhythmia in normal populations of athletes. The problem of ventricular arrhythmias is regarded as extremely important because they are the most frequent cause of sudden cardiac death in athletes. At the other side, ventricular arrhythmias may be a benign feature of "athlete's heart syndrome" [1].

One of the theories of ventricular arrhythmia at athletes relates to large areas of delayed gadolinium enhancement (DGE) in the wall of the LV in Magnetic Resonance Imaging (MRI), sometimes as an incidental finding in athletes presenting with an increase in ventricular premature beats (VPB) or new repolarization abnormalities. Current hypothesis is that these scar regions are the sequalae of subclinical myocarditis. Athletes with such incidental finding, however, seem to have a high likelihood to develop major ventricular arrhythmias during follow-up [2].

The most important studies were performed in Italy. Since 1963 over a 30-year period, more than 125.000 athletes were evaluated in 9 sports medical centers. 3,190 subjects (2.5\%) were disqualified. In $51 \%$ of them the reason for disqualification was of cardiovascular origin, with arrhythmias as the most frequent abnormality [3]. It is estimated that $30 \%$ of athletes do have frequent and complex ventricular tachyarrhythmias on ambulatory Holter electrocardiography. Treadmill tests significantly underestimate the frequency and the grade of arrhythmias [4].

Only 7\% of athletes presenting arrhythmias have features of structural cardiac disease. Cardiovascular abnormalities are significantly more common in athletes with complex ventricular arrhythmias and frequent premature ventricular beats ( $>2000 / 24$ hours). In $30-40 \%$ of them, underlying myocardial disease is present, most frequently arrhythmogenic right ventricular cardiomyopathy, dilated cardiomyopathy, mitral prolapse and myocarditis. Noninvasive cardiac screening including ECG, exercise tests, 2-dimensional and Doppler echocardiography, 24-hour Holter monitoring, and in selected cases, cardiac magnetic resonance imaging allows us to identify potentially pathologic substrate and life-threatening electrical instability [5]. 
Ventricular tachyarrhythmias, identified in elite athletes without cardiovascular abnormalities, are frequent expressions of the "athlete's heart syndrome" (up to 70\%), though they are not related to the presence or magnitude of training-induced LV hypertrophy [6]. Brief deconditioning (5-6 months) usually results in the resolution of arrhythmias in athletes without organic heart disease [7]. Intense endurance training may lead to pathological proarrhythmogenic right ventricular remodeling. It is why among endurance athletes, ventricular arrhythmias most frequently arise from the right ventricle. Usually these arrhythmias, even nonsustained VT, are benign if structural, functional, and electrical abnormalities do not fulfil arrhythmogenic right ventricular cardiomyopathy criteria. Recently, an isolated subepicardial right ventricular outflow tract scar being a substrate for fast VT in high-level endurance athletes has been reported [8]. This kind of arrhythmia can be successfully treated by ablation, giving the athletes the chance for coming back to sports. Differentiation between healthy athletes with benign RVOT arrhythmia and concealed forms of potentially malignant right ventricular cardiomyopathy is difficult. Echocardiographic and CMR measures of right ventricular functions performed during exercise may reveal underlying cardiac disease.

The risk of sudden cardiac death in athletes is 3.5-4.5 times higher than in their counterparts not playing sports $[9,10]$. The risk is enhanced by concealed cardiac entity predisposing to life threatening arrhythmias. Hypertrophic cardiomyopathy and arrhythmogenic right ventricular cardiomyopathy, followed by myocarditis, channelopathies and coronary artery anomalies in young athletes and coronary heart disease in older athletes $(>33 y)$, are the most important causes of sudden cardiac death during effort. Increase of sympathetic activity, electrolyte imbalance, hyper- or hypothermia, dehydration and ischemia are direct triggers for the presence of an organic or electrical substrate. Most arrhythmogenic entities may be detected during preparticipation examination, comprising an ECG which is associated with substantial reduction in cardiovascular mortality. Appropriate precautions including medical treatment, ablation, sympathetic cardiac denervation and/ or ICD implantation and a choice of an adequate sport may reduce the necessity of disqualification.

Among cardiac entities responsible for sudden cardiac death in athletes, ARVC has a special place. No wonder that volume and pressure overload during endurance effort influences pathological right ventricular remodeling in individuals with desmosome mutations causing arrhythmogenic right ventricular cardiomyopathy, the entity being one of the most frequent causes of sudden death in athletes. Indeed, ARVC patients are frequently athletes and arrhythmias occur mainly during athletic activity. Playing sports increases the risk of sudden cardiac death more than fivefold in ARVC patients. Moreover, there is a relationship between a disease's severity and the intensity and duration of exercise. James et al. documented the association of exercise with earlier onset and more severe arrhythmic and structural disease evidence in ARVC mutations carriers [11].
Recently, a hypothesis of sport-dependence acquired form of ARVC was proposed by Heidbuchel and La Gerche [12]. In athletes with ARVC, desmosome mutations are significantly less frequent than in ARVC patients who do not play sports. This susceptibility may come from mutations in unknown genes or combinations of polymorphisms. A recent international expert consensus statement on the treatment of ARVC recommends a restriction from competitive and/or endurance sports, as well as from other athletic activities except for recreational low-intensity sports in all symptomatic and asymptomatic ARVC patients. In healthy gene mutations, a carrier's restriction from competitive sports is recommended $[13,14]$.

Ventricular tachycardia (VT) is uncommon in athletes and frequently occurs in the presence of structural heart disease. VT can occur in the absence of detectable heart disease. Because of the preservation of normal cardiac function, these arrhythmias are generally well tolerated. These ventricular arrhythmias are usually associated with a favorable prognosis in normal individuals, but they could have a clear impact on athletic performance and career [15].

Exercise-induced ventricular arrhythmias raise clinical concerns as a marker of increased risk in the presence of underlying cardiovascular disease. In a recently published paper by a group of recognized Italian and American experts, exercise ECG testing was performed in above 5000 consecutive athletes without heart disease and analyzed for the occurrence of ventricular arrhythmias [16].

Of the 5011 athletes, $367(7,3 \%)$ showed > 1 premature ventricular beat (PVB), including $331(6,6 \%)$ with $<10$ PVBs and $36(<1 \%)$ with more than 10 PVBs and/or $>1$ ventricular couplet and/or $>1$ burst of nonsustained ventricular tachycardia. The 331 athletes with $<10$ PVBs had no restrictions from competitive sports and repeated testing over 3-12 months showed a spontaneous reduction of arrhythmia, including 83/331 with the disappearance of PVBs. The remaining 36 athletes were disqualified from sports because of frequent and/or complex arrhythmias; 23 showed reduction of arrhythmia at 3-12 months and were readmitted to competition. The other 13 athletes with persistent arrhythmias were considered for radiofrequency ablation: six of them were successfully treated with abolition of arrhythmias and permitted to return to competitive sports. No events of cardiovascular disease occurred in the 367 athletes over a follow-up period of $7.4+/-5$ years [16]. Exercise-induced cardiac arrhythmias have been observed in ca $9 \%$ of 109 experienced participants (mean age = 48 years; mean marathon time $249 \mathrm{~min}$; ca $24 \%$ were women) of marathon races in Berlin/Germany. The authors used portable ECG recorders to document cardiac arrhythmias and ST -T segment abnormalities during the race. Altogether in about $17 \%$ of male and women runners, they identified abnormal ECG findings [17].

Ventricular tachycardia is often the cause of implantation of a cardioverter defibrillator (ICD) to prevent sudden cardiac death (SCD). In the case of athletes, this always causes a lot of discussion 
because of problems with continuation of physical activity that is associated with tachycardia and unjustified ICD discharges. Care of the Athletic Heart 2019 (CAH), directed by Matthew Martinez MD, and Jonathan Kim, MD, convened June 20-22 at the American College of Cardiology's Heart House in Washington, DC. Dr. Guru Kowlgi ended the session with a case of bidirectional ventricular tachycardia in a recreational endurance athlete due to catecholaminergic polymorphic ventricular tachycardia. It was a rare moment when the panel and audience (220participants) unanimously agreed that aggressive beta-blocker therapy should be the mainstay of treatment in this disease and that ICDs should only be used if syncope/ventricular tachycardia recurs despite medical therapy [18].

\section{The Usefulness of Sport Heart Rate Monitors in Detecting Ventricular Arrhythmias}

Nowadays, it is difficult to imagine an endurance athlete implementing training plans without a sports heart rate monitor (HRM) [19]. This applies to runners, cyclists or swimmers [20]. Although HRMs are intended for healthy athletes (with sinus rhythm) to implement a training plan, they can "catch" cardiac arrhythmias [21]. HRMs, by assessing the current heart rate, count the main electric field resulting from ventricular contraction. HRMs are not an electrocardiograph and they do not recognize P waves and QRS complexes, so they, read" arrhythmia as a slow or fast heart rate. Whether tachyarrhythmia or bradyarrhythmia is supraventricular or ventricular is not identifiable with HRMs. HRMs with the function of heart rhythm variability (HRV) inform us about regularity rhythm or lack of it, still not allowing to determine what causes the regularity disorder. Single supraventricular and ventricular beats are completely indistinguishable. Athletes struggling with the symptoms of bradyarrhythmia or tachyarrhythmia must undergo professional diagnostics with the need to perform a minimum ECG and Holter ECG $[22,23]$. This also applies to athletes with paroxysmal atrial fibrillation suspicions [24].

\section{Conclusion}

In summary, it should be stated that although HRMs are a great tool to control the intensity of training by controlling the heart rhythm and can accidentally "catch" arrhythmias, their usefulness in the diagnosis of ventricular arrhythmia is limited.

\section{References}

1. Fuchs T, Torjman A, Galitzkaya L, Leitman M, Pilz-Burstein R, et al. (2011) The clinical significance of ventricular arrhythmias during an exercise test in non-competitive and competitive athletes. Isr Med Assoc J 13(12): 735-739.

2. Heidbuchel H (2018) The athlete's heart is a proarrhythmic heart, and what that means for clinical decision making. EP Europace 20(9): 14011411.

3. Pelliccia A, Maron BJ (1995) Preparticipation cardiovascular evaluation of the competitive athlete: Perspectives from the 30-year Italian experience. Am J Cardiol 75(12): 827-829.

4. Minamitami K, Miyagawa M, Kondo M, Kitamura K (1980) The electrocardiogram of professional cyclists. In: Lubich T \& Venerando A
(Eds.), Sports Cardiology p. 315.

5. Biffi A, Pelliccia A, Verdile L, Fernando F, Spataro A, et al. (2002) Long-term clinical significance of frequent and complex ventricular tachyarrhythmias in trained athletes. J Am Coll Cardiol 40(3): 446-452.

6. Biffi A, Maron BJ, Di Giacinto B, Porcacchia P, Verdile L, et al. (2008) Relation between training-induced left ventricular hypertrophy and risk for ventricular tachyarrhythmias in elite athletes. Am J Cardiol 101(12): $1792-1795$

7. Biffi A, Maron BJ, Verdile L, Fernando F, Spataro A, et al. (2004) Impact of physical deconditioning on ventricular tachyarrhythmias in trained athletes. J Am Coll Cardiol 44(5): 1053-1058.

8. Tandri H, Hutchinson MD (2017) Scar-related right ventricular tachycardias in athletes. J Am Coll Cardiol 69(5): 508-510.

9. Corrado D, Basso C, Rizzoli G, Schiavon M, Thiene G, et al. (2003) Does sports activity enhance the risk of sudden death in adolescents and young adults? J Am Coll Cardiol 42(11): 1959-1963.

10. Marijon E, Uy-Evanado A, Reinier K, Teodorescu C, Narayanan K, et al. (2015) Sudden cardiac arrest during sports activity in middle age. Circulation 131(16): 1384-1391.

11. James CA, Bhonsale A, Tichnell C, Murray B, Russell SD, et al. (2013) Exercise increases age-related penetrance and arrhythmic risk in arrhythmogenic right ventricular dysplasia/cardiomyopathy associated desmosomal mutation carriers. J Am Coll Cardiol 62 (14): 1290-1297.

12. Heidbüchel H, La Gerche A (2012) The right heart in athletes. Evidence for exercise-induced arrhythmogenic right ventricular cardiomyopathy. Herzschrittmacherther Elektrophysiol 23(2): 82-86.

13. Maron BJ, Zipes DP, Kovacs RJ (2015) Eligibility and disqualification recommendations for competitive athletes with cardiovascular abnormalities: Preamble, principles, and general considerations: A scientific statement from the american heart association and american college of cardiology. Journal of the American College of Cardiology 66(21): 2343-2349.

14. Corrado D, Wichter T, Link MS, Hauer R, Marchlinski F, et al. (2016) Treatment of arrhythmogenic right ventricular cardiomyopathy/ dysplasia: an international task force consensus statement. Circulation. 133(11): e437-8.

15. Hoogsteen J, Bennekers JH, van der Wall EE, van Hemel NM, Wilde AA, et al. (2004) Recommendations and cardiological evaluation of athletes with arrhythmias: Part 1. Neth Heart J 12(4):157-164.

16. Verdile L, Maron BJ, Pelliccia A, Spataro A, Santini M, et al. (2015) Clinical significance of exercise-induced ventricular tachyarrhythmias in trained athletes without cardiovascular abnormalities. Heart Rhythm 12(1): 7885.

17. Herm J, Töpper A, Wutzler A, Kunze C, Krüll M, et al. (2017) Frequency of exercise-induced ST-T-segment deviations and cardiac arrhythmias in recreational endurance athletes during a marathon race: results of the prospective observational Berlin Beat of Running study. BMJ Open 7(8): e015798.

18. Rao P (2019) Arrhythmias in athletes: Let's make the decision together, care of the athletic heart, 2019.

19. Gajda R, Biernacka EK, Drygas W (2018a) Are heart rate monitors valuable tools for diagnosing arrhythmias in endurance athletes? Scand J Med Sci Sports 28(2): 496-516.

20. Gajda R, Kowalik E, Rybka S, Rębowska E, Śmigielski W, et al. (2019a) Evaluation of the heart function of swimmers subjected to exhaustive repetitive endurance efforts during a 500-km relay. Front Physiol 10: 296.

21. Gajda R, Biernacka EK, Drygas W (2018b) “The problem of arrhythmias in endurance athletes: are heart rate monitors valuable tools for 
diagnosing arrhythmias?", Horizons in World Cardiovascular Research. Nova Science Publishers, New York, USA 15: 1-64

22. Gajda R (2019a) Commotio cordis at athletes-under recognized problem. Res Inves Sports Med 5(3). RISM.000615.2019.

23. Gajda R (2019b) Extreme bradycardia and bradyarrhythmias at athletes. what will technology development bring as a help to diagnosis them? Res Inves Sports Med 5(4). RISM.000617.2019.

24. Gajda R, Biernacka EK, Drygas W (2019b) Atrial fibrillation in athleteseasier to recognize today? Res Inves Sports Med 5(4): RISM.000618.2019.

For possible submissions Click below: 